\title{
ENGEVISTA
}

Página da revista: http://www.uff.br/engevista/seer/

\section{Análise de um retificador monofásico com alto fator de potência utilizando o conversor boost interleaved de duas fases frente às normativas IECiec61000- 3-2 (2009) classe D}

\author{
Analysis of a single-phase rectifier with high factor of use of two-phase boost \\ interleaved converter in compliance with IEC61000-3-2 (2009) class D \\ Israel Divan Lopes da Costa $1^{1}$ \\ Carlos Renato Magalhães Duarte ${ }^{2}$ \\ Diego Santos Gonçalves ${ }^{3}$
}

Resumo: Este artigo objetiva-se a avaliar de maneira prática, a aplicação do conversor boost intercalado no modo pre-regulador de fator de potência, como solução ao problema de distorção harmônica e empobrecimento do fator de potência que os retificadores convencionais associados a filtro capacitivo, interface inicial entre a rede elétrica monofásica ou trifásica e muitos dos sistemas eletrônicos de potência, inserem no sistema elétrico. O baixo fator de potência, baixa eficiência energética, inserção de distorção harmônica e interferência eletromagnética se tornaram hoje os principais distúrbios gerados de maneira indireta pelas cargas que compõe o sistema elétrico de potência. Retificação com alto fator de potência se torna neste contexto, uma obrigatoriedade frente às normativas IEC61000-3-2 e IEEE519. A preocupação com a qualidade da energia elétrica levará a uma nova topologia, que trata da retificação em alto fator de potência utilizando-se da estratégia do PFC Ativo aplicando o conversor boost, e como otimização de eficiência desta estrutura será demostrado posteriormente o conversor em arranjo intercalado - Interleaved $P F C$. Ao analisarmos de maneira prática os resultados obtidos, percebe-se o quão benéfico se torna para o sistema elétrico de potencia, trabalhar-se com correção ativa de fator de potencia integrada a sistemas frontend em equipamentos

eletroeletrônicos. A densidade de potencia de um equipamento pode melhorar significativamente ao se intercalar múltiplos conversores no que se relaciona a minimização dos elementos passivos filtrantes de componentes EMI.

Palavras-chave: PFC; Retificadores; Booster; Interleaved.

\footnotetext{
${ }^{1}$ UNILESTE - Centro Universitário do Leste de Minas Gerais.

${ }^{2}$ UNILESTE - Centro Universitário do Leste de Minas Gerais.

${ }^{3}$ UNILESTE - Centro Universitário do Leste de Minas Gerais.
} 


\begin{abstract}
This article aims to evaluate the practical application of the boost in active PFC mode as a solution to the problem of harmonic distortion and power factor depletion that conventional rectifiers associated with capacitive filter, initial interface between the single-phase electric network or three-phase, and electronic power systems, insert into the electrical system. Low power factor, low energy efficiency, harmonic distortion insertion and electromagnetic interference have now become the main indirectly generated disturbances due to the loads that make up the electric power system. Rectification with high power factor becomes in this context, an obligation in front of the norms IEC61000-3-2 and IEEE519. Concern about the quality of electric power will lead to a new topology, which deals with rectification in high power factor using the strategy of Active PFC applying the boost converter and, how optimization of efficiency of this structure, will be demonstrated later the converter in interleaved arrangement - Interleaved PFC. By analyzing in a practical way the results obtained, one can see how beneficial it is for the electric power system, to work with active power factor correction integrated with front-end systems in electrical and electronic equipment. The power density of equipment improves significantly when interleaving multiple converters in what concerns the minimization of the passive elements filtering of EMI components.
\end{abstract} Keywords: PFC; Rectifiers; Booster; Interleaved. 


\section{Introdução}

Os PFC's (Power Faction Correction) são retificadores com alto fator de potência em suas mais diversificadas topologias de projeto. Tornaram-se uma alternativa aos sistemas retificadores que em sua concepção mais simples apresentam alta distorção harmônica da corrente de entrada em função do transitório de carga do banco de capacitores associado a tais dispositivos. Segundo Rahman et al 2016, os conversores boost configurados como interface inicial entre a rede elétrica tem a capacidade de sincronizar a corrente de entrada do dispositivo com a tensão principal de alimentação, ambas de natureza senoidal, a fim de maximizar a potência real extraída da rede elétrica. A corrente de entrada segue a tensão de entrada em sincronismo, como em uma carga de natureza resistiva, livre de conteúdo harmônico expressivo.

Regidos pela IEC 61000-3-2 de 2009 os equipamentos de baixa potência precisam respeitar um limite de distorção harmônica total em um nível de tensão pré-estabelecido, cabendo assim aos fabricantes viabilizarem seus equipamentos dentro das normas de gerenciamento de energia elétrica. Isso obriga que, em muitos casos, esses equipamentos passem a adotar dispositivos concebidos para adequação de normas e regimentos internacionais. Um desses dispositivos é o conversor boost operando como pré-regulador de fator de potência. O fator de potência apresenta seu valor degradado em função de níveis elevados, na ordem de $100 \%$ de distorção harmônica em virtude de cargas de natureza não linear e altamente reativas. O interleaved PFC de duas fases insere o aperfeiçoamento do dispositivo, onde os estágios de potência operam defasados $180^{\circ}$, trazendo assim, valores desprezíveis das componentes de ripple PWM na corrente de entrada do arranjo, minimizando a interferência eletromagnética e otimizando o desempenho.

Este trabalho fará uma abordagem prática e teórica do conversor boost configurado como pré-regulador de fator de potência $(\mathrm{PFC})$, em solução a problemática de distorção harmônica e empobrecimento do fator de potência que interfaces convencionais utilizando retificação e filtragem capacitiva insere no sistema elétrico.

A validação da proposta apresentada será feita a partir de um protótipo de retificador boost interleaved de 300W que será aferido em laboratório utilizando-se de um analisador de qualidade de energia. Os dados coletados serão cruzados com as normativas IEC61000-3-2 (2009) classe D para equipamentos eletrônicos.

\section{Desenvolvimento}

\subsection{Interface front-end aplicado em Retificadores a diodo}

A interface inicial entre a rede elétrica, monofásica ou trifásica, e os sistemas eletrônicos de potência, é denominada front-end, que tem o propósito de converter a frequência de linha CA em CC. Segundo Mohan (2014), essas interfaces (front-ends), figura 1, podem ser classificadas como retificadores em pontes a diodo, conversores em modo chaveado, em que as 
correntes de linha são de natureza senoidal no fator de potência unitário, objetivo deste trabalho, e conversores a tiristores, em que o fluxo de potência pode ser bidirecional.

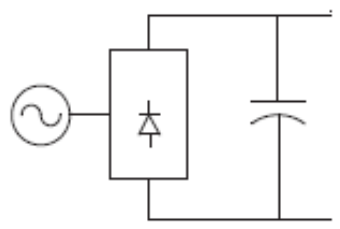

(a)

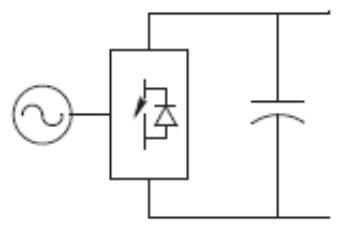

(b)

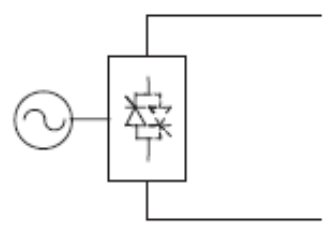

(c)

Figura 1: Front-end de equipamento eletrônico de potencia. a - retificador, b - conversor, c - ciclo conversor.

Fonte: Mohan, (2014) p.97

A Figura 2a apresenta um circuito retificador de onda completa, em que $L_{s}$ é a soma da indutância interna da alimentação pela rede com uma indutância interna, que pode ser colocada em série com a resistência $R_{S}$.

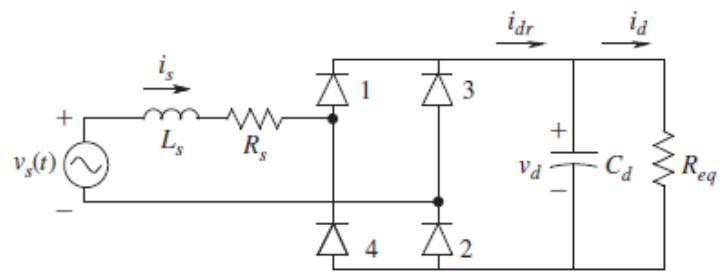

(a)

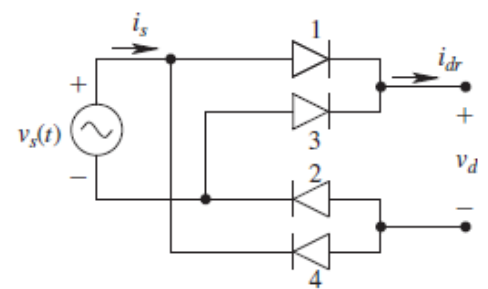

(b)

Figura 2: Retificador a diodo de onda completa em ponte.

Fonte: Mohan, (2014) p. 108

$\mathrm{O}$ circuito retificador anterior apresenta a indutância e a resistência no lado CA, podendo-se ser ignorados. A Figura $2 \mathrm{~b}$ ilustra um circuito que consiste um grupo superior e o outro grupo inferior de diodos. Para que a corrente $i_{d r}$ no lado CC flua, um diodo em cada grupo deverá conduzir.

Nesses sistemas o fluxo de potência é unidirecional, e sem nenhum controle sobre a tensão do barramento CC. São caracterizados por drenarem correntes com formas de onda altamente distorcidas.

Tal arranjo retificador tem característica de empobrecimento do fator de potência em função da falta de linearidade da corrente de entrada em função do transitório de carga em $C_{d}$.

$\mathrm{Na}$ figura 3, parte inferior, fica evidenciada a forma de onda da corrente, drasticamente distorcida em relação à tensão de entrada que tem natureza senoidal, parte superior da figura. 


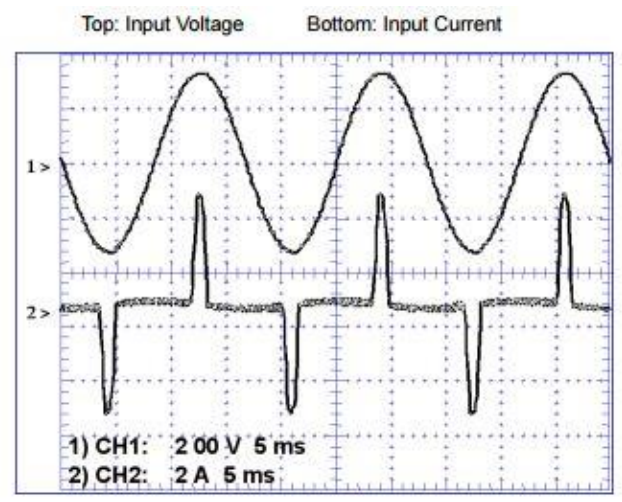

Figura 3: Distorção harmônica da corrente em relação a tensão Fonte: Turchi et al. (2011) p.10

Mohan (2014), afirma que fontes de potência CC em modo chaveado, para atender os limites harmônicos estipulados em norma, devem ser complementados por um circuito de correção de fator de potência.

\subsection{Conversor Boost}

O conversor boost ou step up é caracterizado como uma estrutura elevadora de tensão. A figura 4 exibe de forma conceitual o conversor. O estágio de saída do conversor consiste na carga $R_{o}$, e em um grande capacitor $C_{o}$ de filtragem, usado para minimizar a ondulação na tensão de saída.

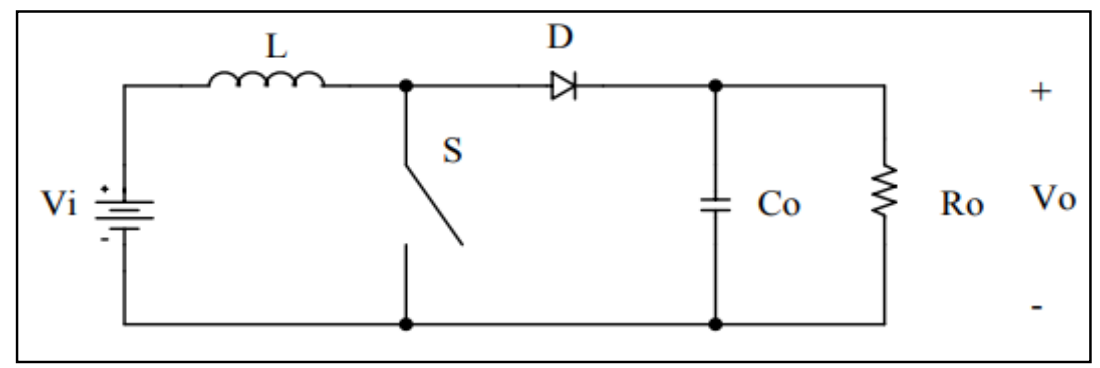

Figura 4: Conversor Boost.

FONTE: Petry, (2011) p. 7

Segundo Petry 2011, o funcionamento do conversor boost, assim como o buck, é regido pelas etapas de condução e corte da chave $\mathrm{S}$.

1ㄹ $\operatorname{Etapa}\left(0, D T_{S}\right): S$ está conduzindo. A fonte $V_{i}$ fornece energia ao indutor. O indutor $L$ é magnetizado.

2a Etapa $\left(D T_{S},(1-D) T_{S}\right): S$ está bloqueado. A fonte $V_{i}$ e o indutor $L$ fornecem energia a saída. O diodo $D$ entra em condução. A tensão na carga aumenta.

A tensão média sobre o indutor deve ser nula, então se define que a função de transferência do conversor evidenciada na equação 2 será: 


$$
\begin{gathered}
\frac{1}{T_{S}} \int_{0}^{D T_{S}} V_{i} d t=\frac{1}{T_{S}} \int_{0}^{(1-D) T_{S}}\left(V_{o}-V_{i}\right) d t \\
\frac{V_{0}}{V_{i}}=\frac{1}{1-D}
\end{gathered}
$$

\subsection{Retificação com alto fator de potência}

A correção do fator de potência $(P F C)$ nas fontes de alimentação é realizada por circuitos passivos ou ativos. De acordo com Lange (2012), a maioria dos retificadores de fator de potência elevado é composta por associações em cascata entre uma ponte retificadora de onda completa a diodos e um conversor CC-CC. Na Figura 5 é evidenciada a configuração mais simples onde um único conversor boost é configurado como PFC ativo, monofásico.

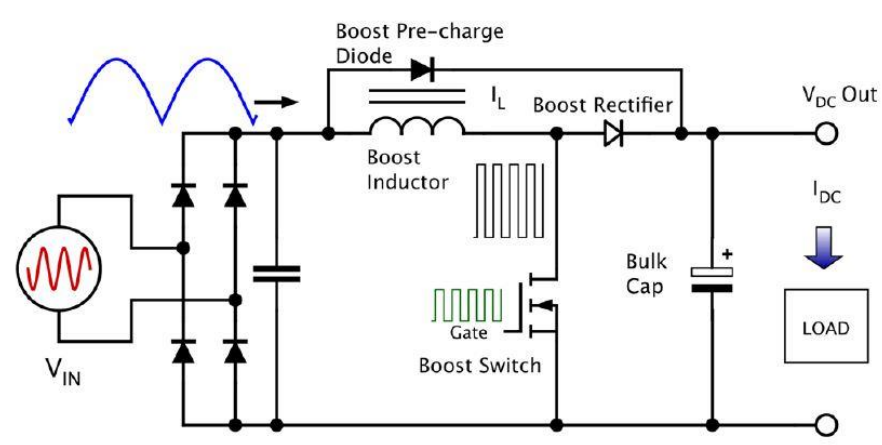

Figura 5: Retificador boost convencional como pre-conversor. Fonte: Rahman et al, (2016) p. 4

Para aplicações de até 1KW a configuração de uma única célula de conversão operando em modo de condução contínua se caracteriza como a solução mais interessante do ponto de vista econômico.

\subsection{Princípio de funcionamento}

Segundo Barbi 2015, o retificador boost (PFC), opera da mesma forma que o conversor boost convencional, porém neste caso a tensão de entrada possui o formato de uma sinusóide retificada. O PFC Monofásico, como mostrado na Figura 6, é frequentemente usado em topologia conversor CC-CC elevador, onde é atribuído entre a alimentação Vs e o capacitor de barramento $\mathrm{CC} C \mathrm{Cd}$. Por meio da modulação por largura de pulso no MOSFET ou IGBT, em uma frequência de chaveamento constante, a corrente $i_{L}$ no indutor $L_{d}$ é formada para obter a forma de onda completa retificada, similar a $V_{s}(t)$. Pela equação 3 definese:

$$
i_{L}(t)=i_{L}|\operatorname{sen} \omega t|
$$




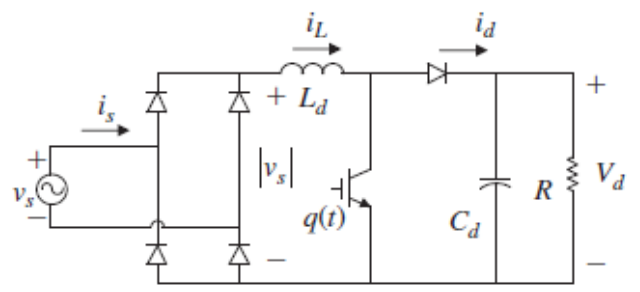

(a)

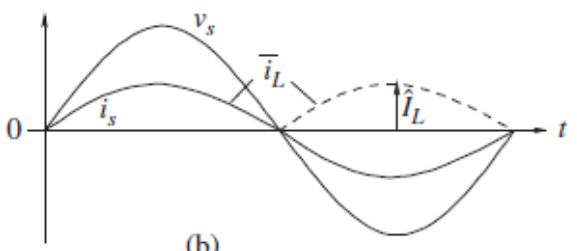

(b)

Figura 6: Circuito PFC e formas de onda.

Fonte: Mohan,(2014) p. 117

A corrente do indutor $i_{L}$ possui grande ondulação na freqüência de chaveamento, que é retirada por um filtro. A corrente de entrada $i_{s}$ na Figura $6 \mathrm{~b}$ se torna senoidal e fica em fase com a tensão de alimentação.

No conversor elevador, é necessário que a tensão no barramento $C C V_{d}$ seja maior do que o pico da tensão de alimentação $V_{s}$ :

$$
V_{d}>V_{s}
$$

Na Figura 7 é mostrado o modelo médio do conversor elevador no modo de condução contínua $C C M$ com $i_{L}>0$ a todo o momento. É desprezada a queda de tensão no indutor e assume-se que a tensão no capacitor seja simplesmente CC. É mostrada a razão de trabalho da chave na equação 7.

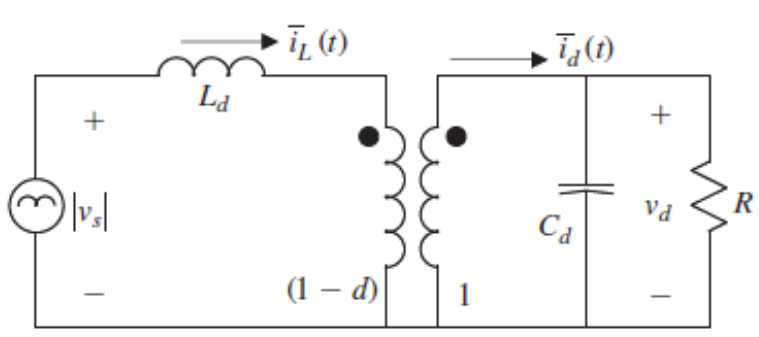

(a)

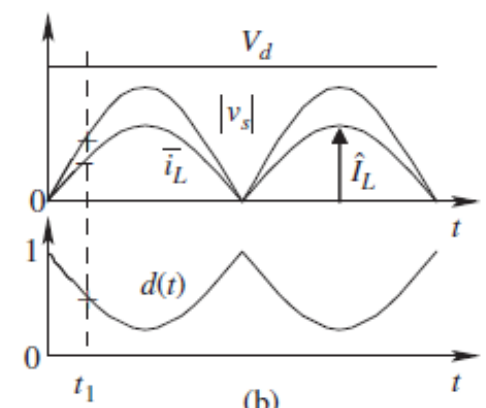

(b)

Figura 7: Modelo Médio em CCM $\left(i_{L}(t)>0\right)$ e formas de onda.

Fonte: Mohan, 2014 p. 117

A relação da razão de transferência de tensão no conversor elevador é definida nas seguintes equações:

$$
\begin{gathered}
\frac{V_{d}}{\left|V_{s}\right|}=\frac{1}{1-d(t)} \\
1-d(t)=\frac{V_{s}|\operatorname{sen} \omega t|}{V_{d}} \\
d(t)=1-\frac{V_{s}|\operatorname{sen} \omega t|}{V_{d}}
\end{gathered}
$$

Sendo:

- $1-d(t)$ : mostra que a chave varia senoidalmente em cada semiciclo da frequiência fundamental, independente do valor da corrente do indutor. 
A corrente que circula no indutor é definida para ser $i_{L}(t)=I_{L}|\operatorname{sen} \omega t|$. Entretanto, a corrente $i_{d}(t)$ pode ser calculada por meio da razão de espiras de um transformador ideal, como demonstrado na equação 8 .

$$
i_{d}(t)=(1-d) i_{L}(t)=(1-d) I_{L}|\operatorname{sen} \omega t|
$$

\subsection{Conversor Intercalado (Interleaved PFC)}

Sistemas PFC intercalados - boost Interleaved PFC consistem na associação de dois ou mais conversores boost em paralelo operando com defasagem no tempo, mas ainda assim, operando da mesma forma conceitual que é abordada nos tópicos 2.3 e 2.4 deste trabalho. Isso traz a enorme vantagem de se poder dividir a potência total do arranjo entre as fases, diminuindo o tamanho físico dos componentes passivos, perdas por condução e cancelamento das componentes PWM em função do chaveamento. Mohan 2014, afirma que, em geral $n$ destes conversores podem ser usados, com fases de operação deslocadas, seguindo a relação de período de chaveamento divido pelo número total de conversores, equação 9.

$$
\text { T por conversor } \quad=\frac{T \text { total }}{\text { numero de fases (conversores) }}
$$

- Onde T é o período da frequência de chaveamento do conversor intercalado.

Segundo O'Loughlin (2013) da Texas Semicondutor, em sistemas de alta potência, associar conversores em modo intercalado se torna uma obrigação para redução das componentes harmônicas de linha, redução do tamanho físico do indutor boost e da corrente de ondulação no capacitor da saída. Ainda segundo ele, isso é conseguido em função do cancelamento que é gerado intercalando os conversores, uma vez que esses operam em defasagem. A figura 8 evidencia o estágio de potência com duas fases intercaladas.

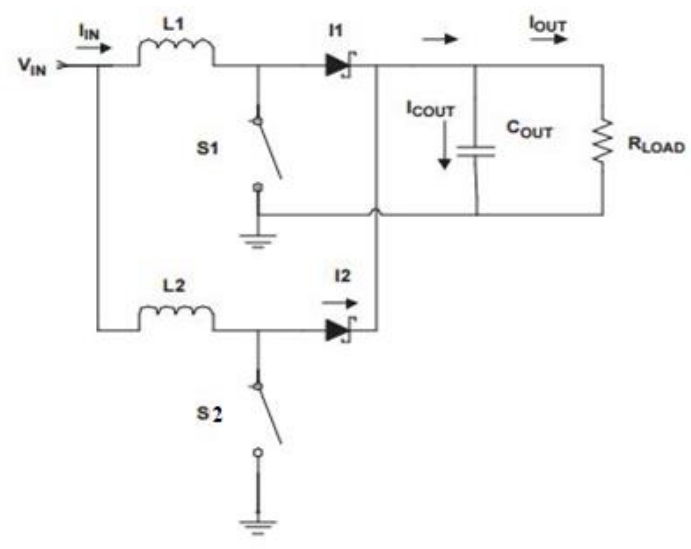

Figura 8: - Arranjo Interleaved $P F C$ de duas fases Fonte: O'Loughlin (2013 p. 2)

A figura 9 evidencia as formas de onda no arranjo intercalado. A defasagem de $180^{\circ}$ entre a fase superior e inferior traz a resultante minimização das componentes harmônicas de chaveamento e ripple de saída, aumentando significativamente a eficiência total do conjunto. 


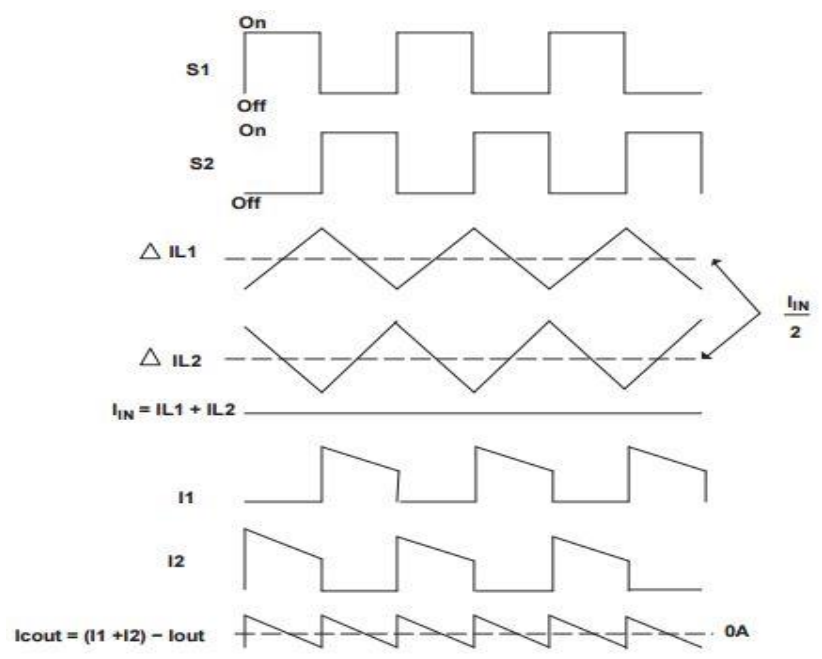

Figura 9: Formas de onda no arranjo interleaved PFC Fonte: O’Loughlin (2013) p. 2

\subsection{IEC61000-3-2 (2009)}

A norma IEC61000-3-2 (2009) estipula limites para as componentes harmônicas em equipamentos com corrente nominal igual ou menor que $16 \mathrm{~A}$ por fase. Estes limites segundo Leão, Sampaio e Antunes 2014, referem-se a equipamentos elétricos e eletrônicos conectados a sistemas de distribuição públicos em corrente alternada, de baixa tensão em 220/380V, 230/400V e 240/415V, operando em 50 ou $60 \mathrm{~Hz}$. Os equipamentos são classificados e agrupados em quatro classes distintas, considerando os critérios do comitê IEC. Tabelas 1 e 2.

Os equipamentos classe $\mathrm{B}$ têm valores harmônicos máximos iguais aos valores definidos para os equipamentos classe A multiplicado por 1,5.

Segundo Leão, Sampaio e Antunes 2014, equipamentos classe C com potência menor que $25 \mathrm{~W}$, conteúdos de terceira e quinta harmônicas da corrente não devem exceder $86 \%$ e $61 \%$ da corrente fundamental. O limite harmônico dos equipamentos classe A e B é constante independente da potência do equipamento desde que seu consumo represente menos que $16 \mathrm{~A}$ por fase. Equipamentos classe C e D têm limites harmônicos proporcionais ao nível de potência do equipamento.

Tabela 1: Classificação de equipamentos (IEC 61000-3-2, 2009).

\begin{tabular}{|l|l|}
\hline Classe A & - Equipamentos trifásicos equilibrados \\
& - Aparelhos domésticos, exceto os classe D \\
& - Ferramentas, exceto as portáteis \\
& - Dimmers para lâmpadas incandescentes \\
& -Equipamentos de áudio \\
& - Todo equipamento não classificado como B,C e D. \\
\hline Classe B & $\begin{array}{l}\text { - Ferramentas Portáteis } \\
\text { - Equipamentos de solda a arco que não seja profissional }\end{array}$ \\
\hline Classe C & - Equipamentos de iluminação para potencias acima de 25W. \\
\hline Classe D & $\begin{array}{l}\text { - Computadores e monitores de PC } \\
\text { - Aparelhos de televisão }\end{array}$ \\
\hline
\end{tabular}


Nota: Potência de entrada $75<\mathrm{P}<600 \mathrm{~W}$. Não há limites para $\mathrm{P}<75 \mathrm{~W}$.

Tabela 2: Limites de Harmônicas de corrente (IEC 61000-3-2, 2009).

\begin{tabular}{|c|c|c|c|c|}
\hline $\begin{array}{l}\text { Harmônicos } \\
{[\text { [n] }}\end{array}$ & $\begin{array}{c}\text { Classe A } \\
{[\text { Corrente em A] }}\end{array}$ & $\begin{array}{c}\text { Classe B } \\
{[\text { Corrente em A] }}\end{array}$ & $\begin{array}{c}\text { Classe C } \\
\text { [\% da corrente } \\
\text { fundamental] } \\
P>25 W\end{array}$ & $\begin{array}{c}\text { Classe D } \\
{[\mathrm{mA} / \mathrm{W}]} \\
75<\mathrm{P}<600 \mathrm{~W}\end{array}$ \\
\hline & & Harmônicos Impares & & \\
\hline 3 & 2.30 & 3.45 & $30 * \mathrm{fp}$ & 3.4 \\
\hline 5 & 1.14 & 1.71 & 10 & 1.9 \\
\hline 7 & 0.77 & 1.155 & 7 & 1.0 \\
\hline 9 & 0.40 & 0.60 & 5 & 0.5 \\
\hline 11 & 0.33 & 0.495 & 3 & 0.35 \\
\hline 13 & 0.21 & 0.315 & 3 & $3.85 / 13$ \\
\hline \multirow[t]{2}{*}{$15 \leq n \leq 39$} & $0.15 \times 15 / \mathrm{n}$ & $0.225 \times 15 / \mathrm{n}$ & 3 & $3.85 / \mathrm{n}$ \\
\hline & & Harmônicos Pares & & \\
\hline 2 & 1.08 & 1.62 & 2 & - \\
\hline 4 & 0.43 & 0.645 & - & - \\
\hline 6 & 0.30 & 0.45 & - & - \\
\hline $8 \leq n \leq 40$ & $0.23 \times 8 / \mathrm{n}$ & $0.345 \times / \mathrm{n}$ & - & - \\
\hline
\end{tabular}

\section{Resultados e discussões}

\subsection{Análise Prática}

A validação dos conceitos aqui abordados é feita avaliando-se através de um analisador de qualidade de energia AEMC Power Pad III 8336 e de um osciloscópio Tektronix TDS1012C-EDU. Todas as correntes foram mensuradas através de dois sensores de efeito hall LEM LA 25-NP. O protótipo boost Interleaved é configurado como pré-regulador de fator de potência, PFC, frente as normativas IEC61000-3-2 (2009) para equipamentos Classe D que caracterizam a maior área de aplicação desta solução em baixa potência. Esse foi construído manualmente seguindo as diretivas de projeto que constam na folha de dados e na nota de aplicação do circuito integrado de controle UCC28060, que contemplam a execução de uma estrutura interleaved PFC operando em modo de condução crítico, com aplicações voltadas à indústria eletroeletrônica. A tabela 3 evidencia os parâmetros de projeto do conversor e as figuras 10, 11 e 12 demonstram o esquema elétrico parcial, protótipo montado e a bancada de testes. Como chaves foram utilizadas dois mosfets IRF840 e dois diodos ultrarrápidos MSR1560. Os indutores foram construídos com núcleo de liga metálica Sendust. 
ISSN: $1415-7314$

ISSN online: $2317-6717$

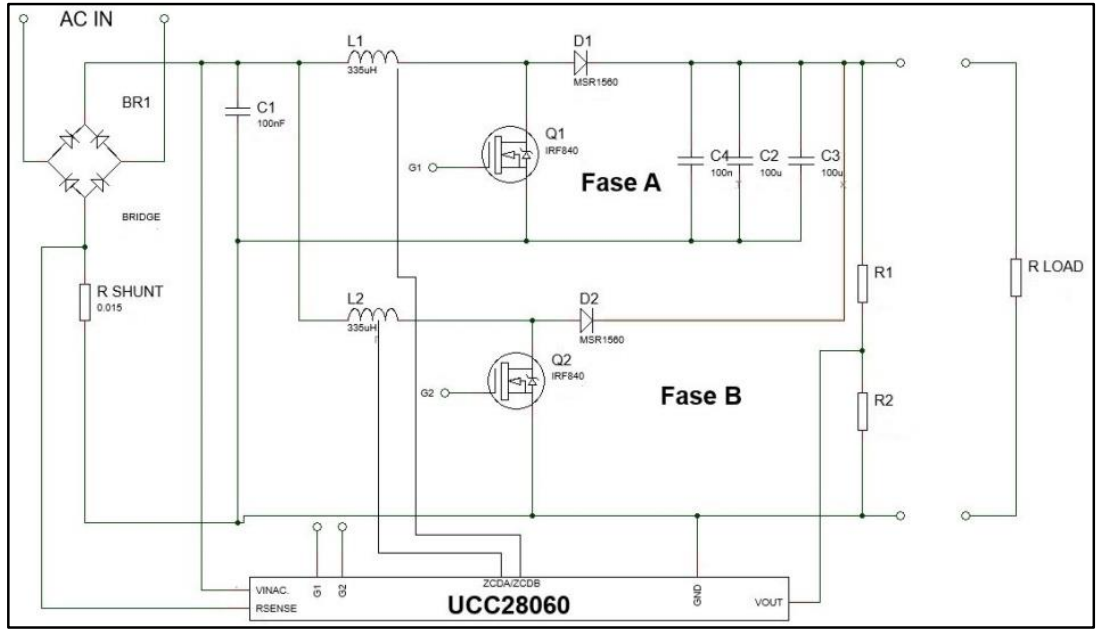

Figura 10: Esquema elétrico parcial do protótipo construído Fonte: Os autores, 2018.

Tabela 3 - Parâmetros de dimensionamento do conversor

\begin{tabular}{|c|c|c|}
\hline V IN & Tensão de Entrada AC RMS & $85 \sim 265 \mathrm{~V}$ \\
\hline V OUT & Tensão de Saída DC & $390 \mathrm{~V}$ \\
\hline f Linha & Frequência de linha & $47 \sim 63 \mathrm{~Hz}$ \\
\hline pf min & Fator de Potencia mínimo & 0.90 \\
\hline POUT max & Potencia de Saída Máxima & $300 \mathrm{~W}$ \\
\hline П min & Rendimento mínimo & $92 \%$ \\
\hline fsw & Frequência de Chaveamento & $45 \mathrm{Khz}$ \\
\hline
\end{tabular}

Fonte: Texas Instruments, UCC28060 Natural Interleaving - Adaptado (2007 p.24)

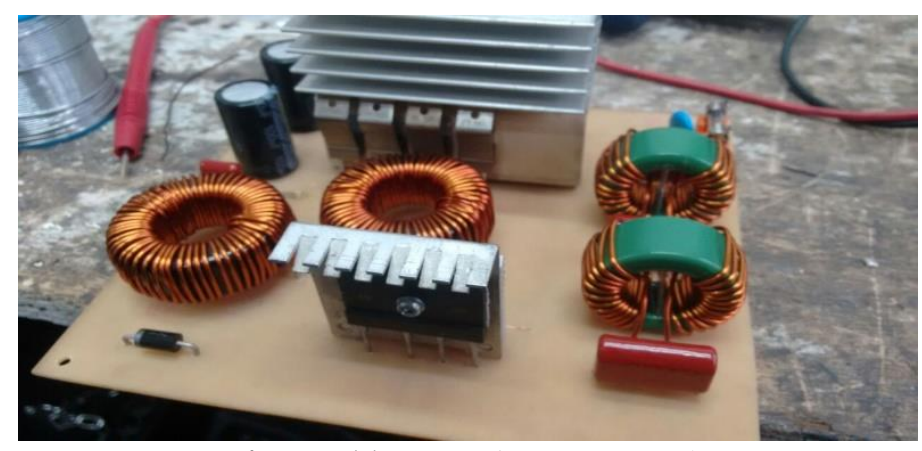

Figura 11: Protótipo construído

Fonte: Os autores, 2018.

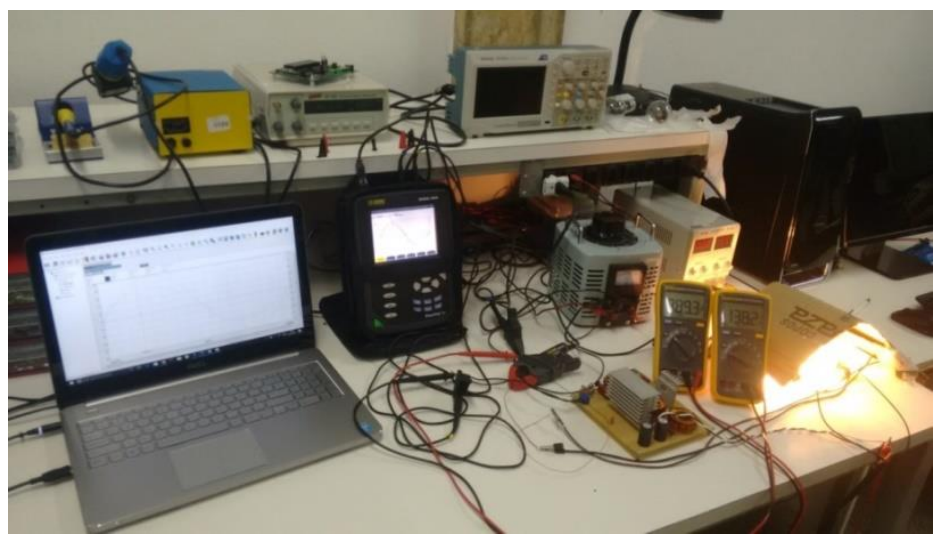

Figura 12: Montagem prática em laboratório

Fonte: Os autores, 2018. 
Os resultados foram coletados em tempo real, através do software Dataview, que compõe o analisador em interação com o computador. Um arranjo de $100 \mathrm{~W}$ de potência composto por lâmpadas incandescentes serviram como carga na saída do conversor. A abordagem inicial desta análise é feita sobre as formas de onda da tensão e corrente na entrada do conversor. A figura 13 exibe a componente de corrente distorcida em relação à tensão de entrada senoidal antes da habilitação do circuito de controle UCC28060.

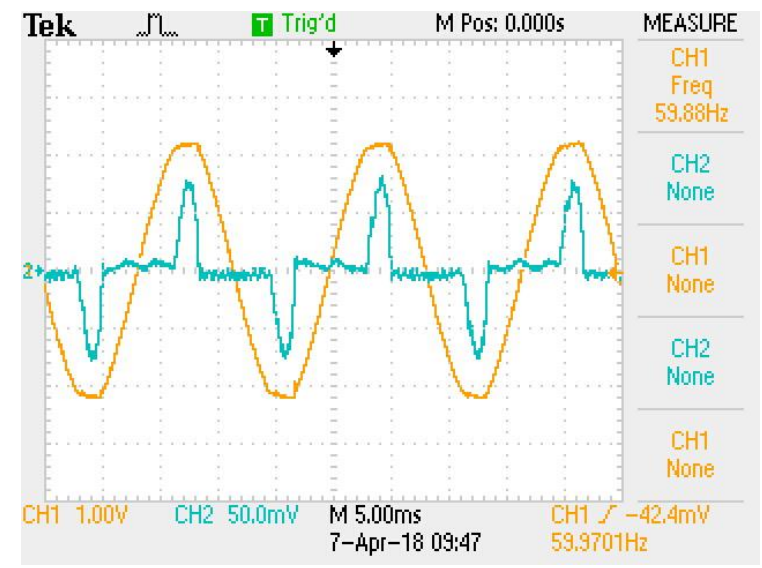

Figura 13: Formas de onda -Tensão e Corrente de entrada do conversor (Tensão em Amarelo. Corrente em azul).

Fonte: Os autores, 2018.

A seguir, a figura 14, após a habilitação do circuito de controle o conversor passa a seguir a referência senoidal replicando em sua entrada uma forma de onda de corrente com mesma natureza da tensão que alimenta o conjunto. As componentes se apresentam com defasamento nulo evidenciando a maximização de potência ativa e fator de potência próximo a unidade.

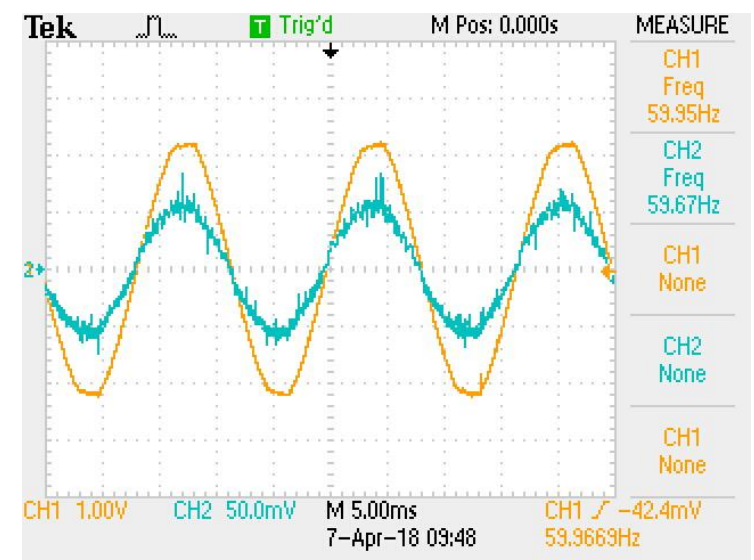

Figura 14: Formas de onda - Tensão e Corrente de entrada do conversor pós habilitação do circuito de controle.

Fonte: Os autores, 2018.

A natureza do fator de potência próxima a unidade e os respectivos valores de potência são apresentados a seguir, capturados através do analisador AEMC 8336, figuras 15 e 16. 


\begin{tabular}{|c|c|c|c|c|c|c|c|}
\hline Channel & Col... Latest & Cursor IIN & Cursor AVG & Cursor MAX & Window MIN & Window AVG & Window MAX \\
\hline PFI & 0,092 & 0,004 & $.0,74$ & $\cdot 0,076$ & 0,092 & 0,845 & 0,993 \\
\hline PFT & 0,992 & 0,004 & $.0,74$ & $\cdot 0,676$ & 0,092 & 0,845 & 0,993 \\
\hline $\operatorname{Cos} \varphi 1$ (DPF) & 0,094 & $\cdot 0,985$ & $-0,985$ & $\cdot 0,985$ & $-0,998$ & 0,871 & 0,997 \\
\hline $\cos \varphi T$ (DPF) & 0,994 & 0,093 & 0,093 & $-0,993$ & 1 & 0,872 & 1 \\
\hline
\end{tabular}

Figura 15: Fator de potência via Dataview

Fonte: Os autores, 2018.

\begin{tabular}{|c|c|c|c|c|c|c|c|}
\hline Channel & Col... Latest & Cursor MIN & Cursor AVG & Cursor MAX & Window MIN & Window AVG & Window MAX \\
\hline $\mathrm{P} 1(\mathrm{~W})$ & $106 \mathrm{~W}$ & $106 \mathrm{~W}$ & $107 \mathrm{~W}$ & $107 \mathrm{~W}$ & $-26,09 \mathrm{~kW}$ & $3,675 \mathrm{~kW}$ & $52,02 \mathrm{~kW}$ \\
\hline PT $(W)$ & $106 \mathrm{~W}$ & $106 \mathrm{~W}$ & $107 \mathrm{~W}$ & $107 \mathrm{~W}$ & $-26,09 \mathrm{~kW}$ & $3,675 \mathrm{~kW}$ & $52,02 \mathrm{~kW}$ \\
\hline $\mathrm{P} 1 \mathrm{DC}(\mathrm{W})$ & $\cdots$ & $\cdots$ & $\cdots$ & $\cdots$ & $\cdots$ & $\cdots$ & $\cdots$ \\
\hline PT DC (W) & $\cdots$ & $\cdots$ & $\cdots$ & $\cdots$ & $\cdots$ & $\cdots$ & $\cdots$ \\
\hline Q1 (var) & -12 var & -13 var & -11 var & -10 var & $-4,852 \mathrm{kvar}$ & -292 var & 2,889 kvar \\
\hline QT (var) & -12 var & -13 var & -11 var & -10 var & $-4,852$ kvar & -819 var & 2,889 kvar \\
\hline D1 (var) & 7 var & 58 var & 60 var & 61 var & O var & 1,137 kvar & $35,52 \mathrm{kvar}$ \\
\hline DT (var) & 7 var & 58 var & 60 var & $61 \mathrm{var}$ & 0 var & $3,23 \mathrm{kvar}$ & 35,52 kvar \\
\hline
\end{tabular}

Figura 16: Potências via Dataview

Fonte: Os autores, 2018.

O consumo de 106W de potência ativa evidencia uma eficiência de $94 \%$ do conversor. A figura 17 evidencia as componentes PWM nos gates dos Mosfets em regime estacionário. O modo de operação com defasagem de 180 (graus) caracteriza o conversor intercalado de duas fases.

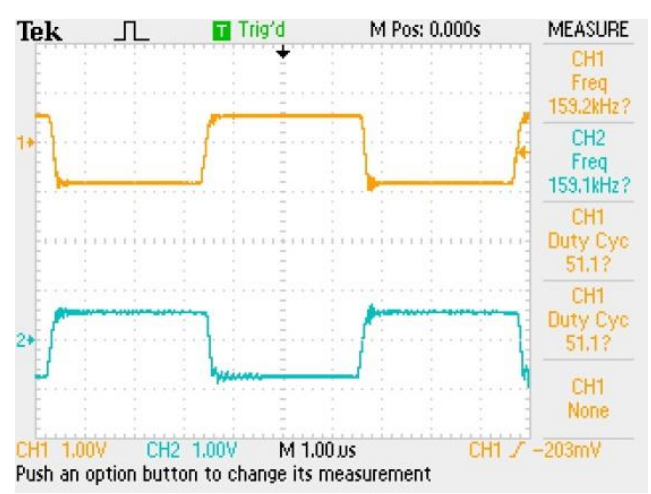

Figura 17: Formas de Onda nos gates dos Mosfets da fase A e fase B.

Fonte: Os autores, 2018.

Por meio da figura 18 pode-se visualizar o ciclo de carga do indutor da Fase A em função da razão cíclica, duty cycle, do Mosfet que compõe esta fase, característica do conversor Boost, onde durante o intervalo de condução D o indutor se carrega e durante o intervalo 1-D, chave em corte, esse se descarrega sobre a carga. 


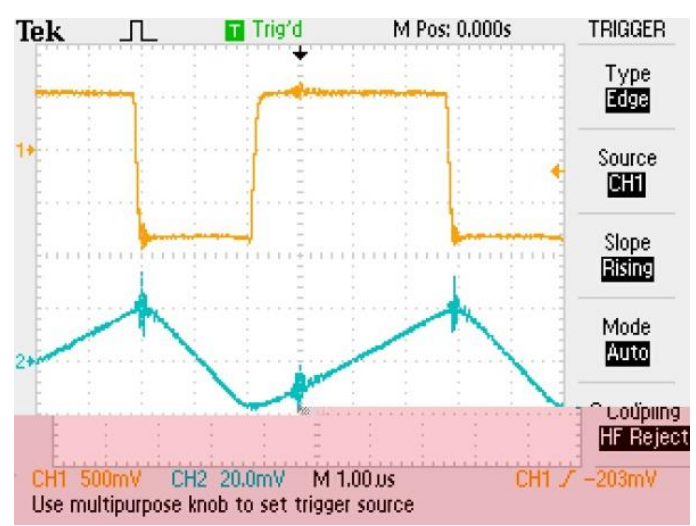

Figura 18: Ciclo de carga e descarga do indutor da fase A em função do ciclo de trabalho do Mosfet.

Fonte: Os autores, 2018.

A figura 19 evidencia o ciclo de carga e descarga dos indutores das fases A e B. A figura 20 demonstra a minimização ripple de corrente na entrada do conversor em comparação com a corrente no indutor da fase B.

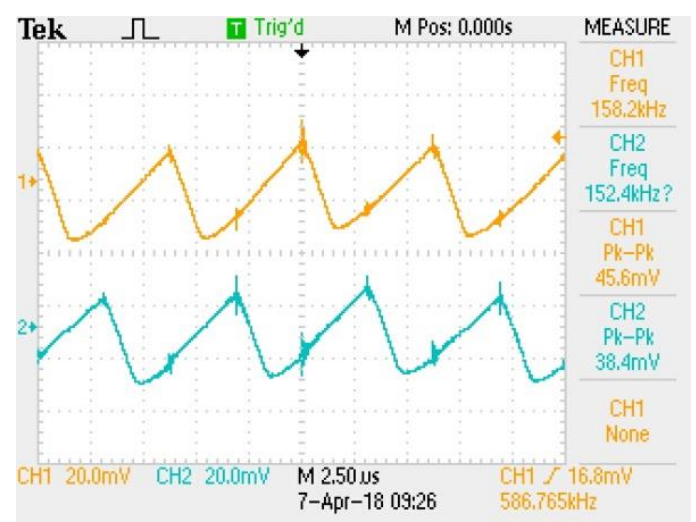

Figura 19: Formas de Onda de corrente nos indutores das fases A em amarelo e fase B em azul. Fonte: Os autores, 2018.

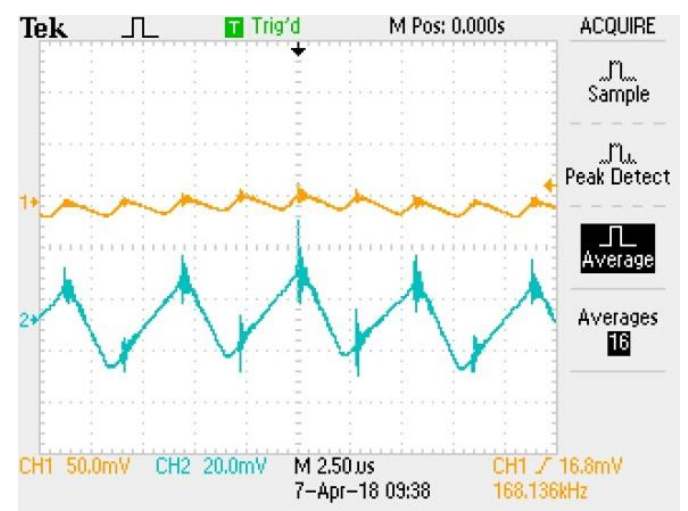

Figura 20: Formas de Onda da corrente de entrada em amarelo e da corrente do indutor Fase B. Fonte: Os autores, 2018.

Por critério de norma o retificador em teste é classificado como classe D dentro da IEC61000-3-2 (2009). Esta norma estipula um limite de 3,40 mA/W de terceira harmônica e $1,90 \mathrm{~mA} / \mathrm{W}$ de quinta harmônica para potências compreendidas entre $75 \mathrm{~W}$ e $600 \mathrm{~W}$. A tabela 4 traz o os limites estipulados pela IEC61000-3-2, (2009) para uma carga classe D com potência 
igual à mensurada no protótipo com conteúdo até a trigésima nona harmônica. A figura 21 exibe o espectro harmônico da corrente de entrada do conversor em teste, mensurado através do analisador de qualidade de energia.

Tabela 4: Parâmetros de dimensionamento do conversor

\begin{tabular}{|c|c|}
\hline $\begin{array}{c}\text { Ordem da } \\
\text { Harmônica } \\
\mathrm{n}\end{array}$ & $\begin{array}{c}\text { IEC61000-3-2 } \\
\text { Dimites para equipamentos classe } \\
\text { potência de protótipo) }\end{array}$ \\
\hline 3 & $360,4 \mathrm{~mA}$ \\
\hline 5 & $201,4 \mathrm{~mA}$ \\
\hline 7 & $106 \mathrm{~mA}$ \\
\hline 9 & $53 \mathrm{~mA}$ \\
\hline 11 & $37,1 \mathrm{~mA}$ \\
\hline 13 & $31,4 \mathrm{~mA}$ \\
\hline $15 \leq \mathrm{n} \leq 39$ & $27,2 \leq \mathrm{n} \leq 10,46 \mathrm{~mA}$ \\
\hline
\end{tabular}

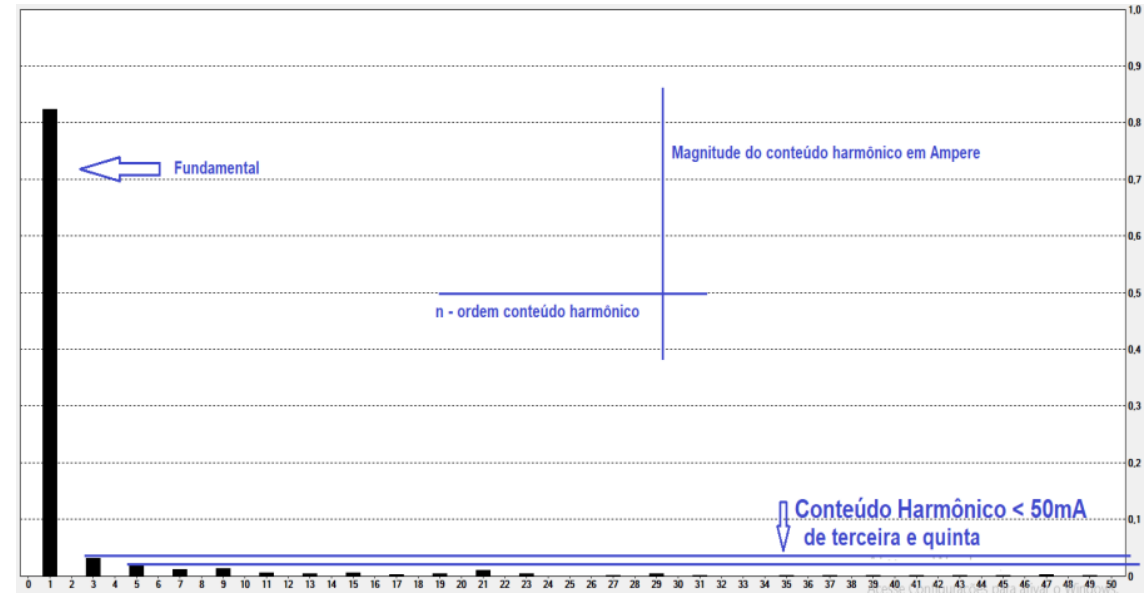

Figura 21: Espectro harmônico da corrente

\subsection{Discussão dos resultados}

O objetivo deste trabalho contemplou a análise prática do conceito de correção do fator de potência de uma estrutura retificadora. Os resultados em laboratório comprovaram a eficiência e funcionalidade desta estrutura utilizada para o propósito especificado. A distorção harmônica ficou abaixo de $2 \%$, a corrente de entrada foi conformada em natureza senoidal, e, em fase com a tensão AC de entrada do arranjo retificador. O fator de potência da ordem de 0,998 comprova que o objetivo do trabalho em questão foi alcançado. A estratégia de intercalar dois conversores trouxe a vantagem de se minimizar o ripple de alta frequência na entrada do conversor, minimizando assim a densidade do filtro de entrada requerido para um conversor fase simples de mesma potência. Sob teste o conversor apresentou consumo de $106 \mathrm{~W}$, que determina um limite de 360,4 mA em terceira harmônica e 201,4 mA em quinta harmônica. Na figura 20 foi possível visualizar que o conteúdo de terceira e quinta harmônicas esteve abaixo de $50 \mathrm{~mA}$, e proporcionalmente todas as demais harmônicas estiveram muito abaixo do limite 
estabelecido em norma, evidenciando a eficácia da técnica de retificação em alto fator de potência através do conversor Boost.

\section{Conclusão}

A utilização de estratégias de correção de fator de potência a nível eletrônico tornouse uma obrigação a ser seguida por grandes corporações e fabricantes de dispositivos eletrônicos, em seus mais diversificados segmentos, e que se utilizam de técnicas de conversão estática para gerenciamento de potência em respeito a normativas internacionais como a IEEE, ANSI, e IEC. Um número expressivo de produtos eletrônicos fabricados devem cumprir requisitos normativos internacionais de emissões e imunidade às implicações em nível da qualidade de energia. Verificou-se por meio da análise dos resultados o quão positivo representa para o sistema elétrico a técnica de retificação em alto fator de potencia integrada a aparelhos eletroeletrônicos. Estas representam um número expressivo de cargas que compõem um sistema elétrico. Com a preocupação crescente dos distúrbios e os efeitos provocados por cargas não lineares nos sistemas de energia, pesquisadores tem buscado aprofundamento em estudos e pesquisas, com finalidade de conhecer a magnitude, características e técnicas de otimização dos fenômenos inerentes a estas cargas. Limitações em termos de componentes harmônicas na corrente e consequentemente na tensão, devem ser impostas com o objetivo de se zelar pela qualidade de energia. A maximização do fator de potencia observada através da técnica empregada neste trabalho trouxe como benefício otimização em eficiência e qualidade de energia quando integrada a um dispositivo eletroeletrônico.

\section{Referências}

BARBI, I. Retificadores Monofásicos com Correção Ativa do Fator de Potência Empregando o Conversor Boost. Santa Catarina: UFSC, 2015.

IEC61000-3-2 Electromagnetic compatibility (EMC) - Part 3-2: Limits - Limits for harmonic current emissions (equipment input current $\leq 16$ A per phase), 2009, p.8. Disponível em: $\langle w w w . i e c . c h>$.

LANGE, A. B., Retificador PFC Monofásico bridgless - Três níveis de alto desempenho. 2012. 232 f. Tese de Mestrado. Universidade Federal de Santa Catarina, Florianópolis, 2012.

LEÃO, R. P. S., SAMPAIO, R. F., ANTUNES, F.L. M. Harmônicos em Sistemas Elétricos. 1. Ed. Rio de Janeiro: Elsevier Editora Ltda, 2014. 354 p.

MOHAN, N. Eletrônica de Potência. Curso Introdutório. São Paulo: LTC, 2014. 270 p.

O'LOUGHLIN, M. 350-W, Two-Phase Interleaved PFC Pre-Regulator Design Review. TEXAS INSTRUMENTS. Texas, 2013.

PETRY, C. A., Introdução aos conversores CC-CC. Instituto de Eletrônica de potência Universidade Federal de Santa Catarina. Florianópolis, 2001.

RAHMAN, S. A., STUCKLER, F., SIU, K., PFC Boost Converter design guide. INFENION. Singapore, 2016.

TEXAS INSTRUMENTS. UCC28060 Natural InterleavingTM DUAL-PHASE TRANSITIONMODE PFC CONTROLLER. 
ISSN: $1415-7314$

ISSN online: $2317-6717$

Disponível em: <http://www.ti.com/lit/ds/symlink/ucc28060.pdf>. Acesso em 15 nov. 2017.

TURCHI, J., DALAL, D., WANG, P., JENCK, L. Power Factor Correction (PFC) Handbook. Choosing the high power fator Controller Solution - HBD853/D. Rev. 5. ON SEMICONDUCTOR, 2011. 